\title{
Survey system for life styles of the elderly and to identify the consumption of food and drink
}

\author{
A. Sawada ${ }^{1}$, T. Oyabu ${ }^{1}$, S. Seto ${ }^{2} \&$ T. Katsube ${ }^{3}$ \\ ${ }^{1}$ Graduate School of Regional Economic Systems, \\ Kanazawa Seiryo University, Japan \\ ${ }^{2}$ Kinjo University, Japan \\ ${ }^{3}$ Faculty of Engineering, Saitama University, Japan
}

\begin{abstract}
From the viewpoint of welfare costs, it is necessary to survey whether older people eat, drink and live their daily lives in safety. It is especially desired that an older person drinks green tea and/or coffee routinely and autonomously takes meals, namely breakfast, lunch and supper. In addition, it is better for an older person to eat fruits such as oranges and apples to prevent colds, Alzheimer's disease and other illnesses. In this study, a system to recognize the eating of a meal, the drinking of a beverage and the eating of a fruit was constructed using odor sensors. Three metal oxide odor sensors were adopted for this system. The system can identify behavior by analyzing characteristics of odor sensors. As for the results, the system recognizes this behavior successfully and also detects the level of fatigue of an older person. A high correlation coefficient (over 0.85) was obtained between the degree of fatigue due to bodily sensations and the output of the sensor. The sensor was highly sensitivity to hydrogen gas. When humans are fatigued or stressed, their exhaled breath contains hydrogen at high concentrations. Discriminating analysis was used to identify the kind of fruits eaten and teeth brushing behavior from odor sensor outputs.

Keywords: odor sensor, sensory system, aged people, welfare system.
\end{abstract}

\section{Introduction}

In Japan, birthrate is declining and the population is aging. Therefore, the labor force and manufacturing capabilities are also decreasing. Other advanced industrial countries are in the same situation. The fees for pensions and medical 
treatment for the elderly are more than $1.0 \times 10^{6}$ million yen per year. The cost of the self-governing body has exerted additional pressure upon the fees. It is important to keep various costs down for the elderly with good services. It is necessary to consider various ideas and results from many branches of study to find reasonable means of settling issues arising from these subjects.

In this study, we examined whether the health condition and life pattern of the elderly can be identified by detecting breath odors. The breath odor changes according to human behavior, namely taking a meal, brushing teeth and drinking beverages. It would be very effective if a system could survey the patterns of daily life and some of diseases of the elderly. We developed a breath odor sensing system for this purpose. Humans generate strong breath odors when they have stress and are fatigued. In this paper, breath odor is defined as the odor generated from the human mouth. Breath odor is also generated when teeth are brushed. This is not bad breath. The main odor is due to menthol when teeth are brushed. On the other hand, breath odor decreases briefly when the subject drinks water, tea or milk.

In Japan, apples and oranges are very familiar to the general person, and these fruits are harvested in the greatest number. They are also effective to keep humans healthy. The Ministry of Agriculture, Forestry and Fisheries of Japan is recommending that fruits should be eaten everyday. Three metal oxide gas sensors were adopted to detect breath odor. This type of sensory system is very popular [1-5]. Daily patterns in the sensor outputs were examined, and the identification of the fruit eaten, and the relationship between the grade of fatigue and sensor output were examined. The system was constructed to survey the life style and fundamental behavior of a solitary elderly person [6]. In addition, the system could potentially identify diseases of the elderly in near future.

\section{Experimental}

Breath odor is generated according to the kind of meal, stress, teeth brushing and when amount of sputum is low. Usually, the breath contains mainly hydrogen and methane at a ratio of approximately 9:1. The percentage of hydrogen in exhaled air increases as the subject experiences stress. The offensive odor substances in the expiration are chiefly ammonia, amines, hydrogen sulfide, indoles and methyl mercaptan.

Breath odor is composed of mainly reducing gases. Therefore, the system adopts three metal oxide gas sensors that are highly sensitive to the reducing gases. The material of two sensors is tin oxide and the third is indium oxide. These three sensors are referred to as sensor 1,2 and 3. Each sensor is connected to a load resistance and dc supply is applied to the circuit. The voltage at both ends of the resistance is called the sensor output. The output increases as the grade of breath odor becomes higher. When the sensor output is at high voltage, the resistance of the sensor is low. A photograph of the three sensors is shown in Figure 1. The sensors are installed on the reverse side of the lid of a glass bottle. The three sensor-outputs are directed to a mobile computer. The program for logging data was developed using $\mathrm{C}$ as the programming language. 
A photograph of the system is shown in Figure 2. The three sensors are incorporated into a stuffed doll and exhaled air is introduced into the doll using a small motor in later units. However, a glass bottle was adopted in this experiment to carry this out easily. The stuffed doll was not used in the experiments. The stuffed doll generally has a comforting effect on human beings.

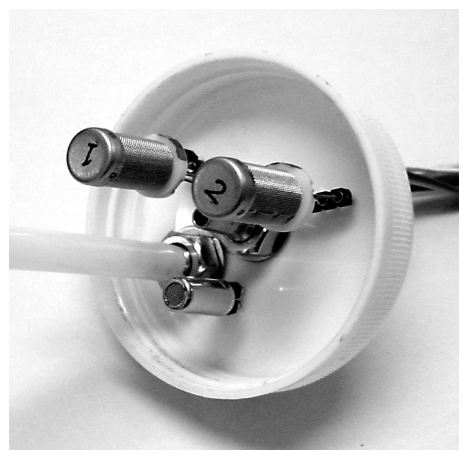

Figure 1: $\quad \begin{aligned} & \text { Three adopted } \\ & \text { sensors installed }\end{aligned}$ on the lid of the experimental bottle.

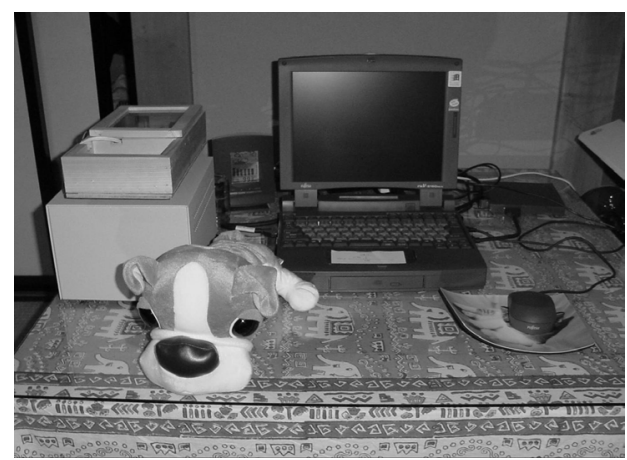

Figure 2:

Experimental system for detecting breath odor.

Eating fruit, brushing teeth and the grade of fatigue are identified using sensor outputs. The glass bottle has an entry and exhaust vent. Its capacity is $150 \mathrm{ml}$. The measurement of breath odor was carried out as follows. The offset level of each sensor was measured for 30 seconds and the breath was introduced for 5 seconds. The breath also ran out via the vent at the same time. The sensor output increased according to the odor grade, and the characteristics could be approximated using an exponential function. The difference between the offset level and the output value at 120 seconds was adopted as the real output for analysis. The experiment was over in 120 seconds.

\section{Results}

\subsection{Daily fluctuation of sensor output}

The outputs of the sensors for $40 \mathrm{ppm}$ of ethyl alcohol are shown in Figure 3. Sensors 1 and 2 ( $\mathrm{S} 1$ and $\mathrm{S} 2$ ), which are made of tin oxide, have a high sensitivity. Sensor 3 (S3), which is made of indium oxide, has a low sensitivity. Examples of the odor sensor outputs for exhaled air are shown in Figure 4. The characteristics were measured after the subject ate Chinese noodle soup. The outputs of sensors 1 and 2 are high because the soup contains many odors. The three sensor outputs for each behavior are shown in Figure 5 as a bar chart. In the figure, the bars for the three sensors were high at breakfast, lunchtime and 

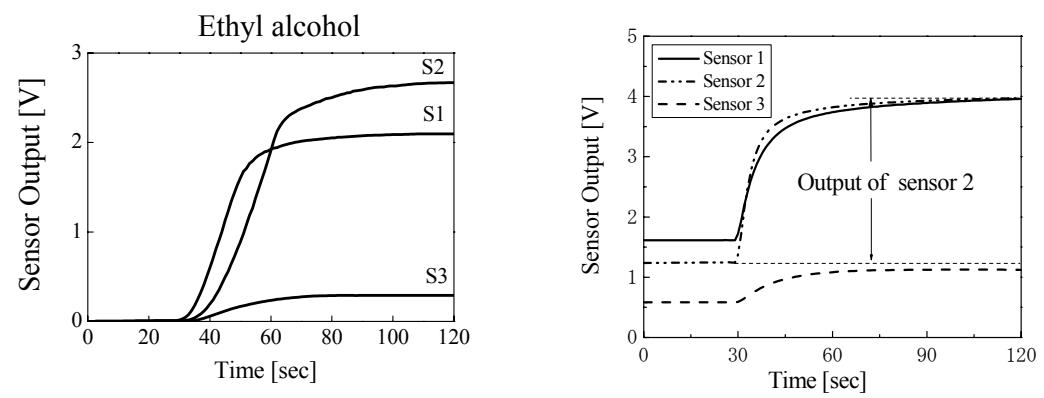

Figure 3: Characteristics of the Figure 4: Characteristics of the three sensors for 40 ppm of ethyl alcohol. three odor sensors for expiration after taking Chinese noodle soup.
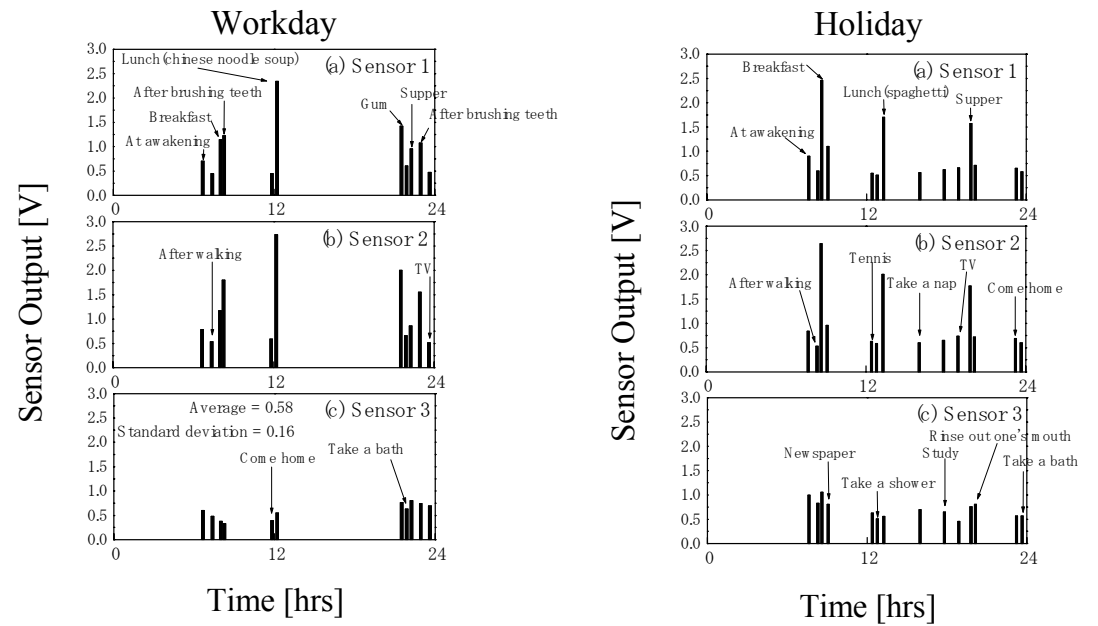

Figure 5: Daily patterns of the Figure 6: Daily patterns of the three sensor outputs displayed on bar graphs. three sensor outputs displayed on bar graphs. The subject did not brush his teeth.

supper. The outputs of sensors 1 and 2 were higher than that for sensor 3 . The output of sensor 3 did not change greatly due to meals. The subject was 54 years old. The outputs of sensors 1 and 2 also increased when the subject chewed gum containing menthol. When the subject ate soup, for example miso soup or noodle soup, the sensor outputs increased. Various kinds of stock are used in noodle soup and miso soup. The flavor is increased by these stocks. The average and standard deviations of sensor 3 output in Figure 5 are 0.58 and 0.16 . The data 
were collected on a workday. The same characteristics were also measured on a holiday, as shown in Figure 6. Teeth were not brushed during the day. The experiment was carried out to remove the influence of teeth brushing. The subject ate a ham sandwich, fresh vegetables and yoghurt at breakfast. The outputs of sensors 1 and 2 are also high and the characteristics are similar to Figure 5. Sensor 3 output fluctuates less than the outputs of sensors 1 and 2 . The averages (ave), standard deviations $(s)$ and coefficients of variation $(c v)$ of the three sensor outputs adopted are summarized in Table 1. The $c v$ is derived by the equation: $s$ / age $\times 100$ [\%]. The values of sensors 1 and 2 show almost the same tendency, while the value of sensor 3 is fairly different to those of sensors 1 and 2.

Taking a meal is important and fundamental behavior for humans, and especially breakfast and supper are important to maintain a healthy condition. We adopted a new coefficient to identify the normal grade of nutrition. The coefficient is introduced by the following calculation. Both sensor 1 outputs for breakfast (mab) and supper (mas) are added, the value is divided by two, and then multiplied 100. The system collected 14 values for 14 days. The data are indicated on a histogram in Figure 7. In the figure, the average of the coefficients is 6.9 and $s$ is 3.4. The coefficient exceeded 12 when the subject ate garlic. We can identify that the subject has normal eating habits when the coefficient is close to the average.

\begin{tabular}{|c|c|c|c|}
\hline \multirow[t]{2}{*}{ Table 1} & \multicolumn{3}{|c|}{$\begin{array}{l}\text { Average (ave), } \\
\text { standard deviation }(s) \\
\text { and coefficient of } \\
\text { variation }(c v) \text { of the } \\
\text { three sensor outputs. }\end{array}$} \\
\hline & $\mathrm{S} 1$ & S2 & S3 \\
\hline ave & 0.94 & 1.00 & 0.71 \\
\hline$s$ & 0.58 & 0.65 & 0.18 \\
\hline$c v$ & 62 & 65 & 25 \\
\hline
\end{tabular}

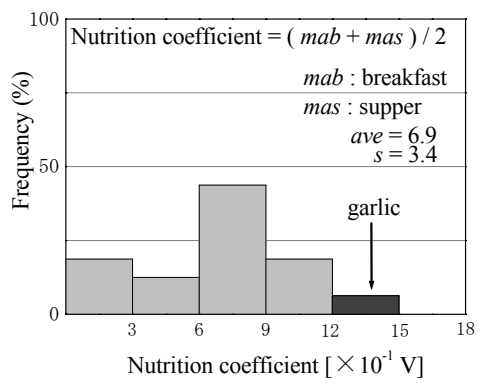

Figure 7: Histogram of sensor 1 output for breakfast and supper.

\subsection{Sensor outputs at awakening and after walking}

Humans generate various odors from their body as they age, and the odor grade becomes high. Breath odor grade also becomes higher. It may be possible to detect some diseases by examining breath odor at awakening. It is an odor for an inactive hour. The sensor output after a walk in the morning is considered as an offset level on the day. The system can recognize progress of disease or of a disorder of body by understanding breath odor characteristics statistically. The histograms of sensor outputs at awakening and after walking in the morning are shown in Figure 8. Figure (a) shows the histogram for sensor 1 output, (b) for sensor 2, and (c) is for sensor 3. The histogram at awakening is displaced toward 
the right and the histogram after walking is displaced toward at left side. Namely, the breath odor grade decreases as a result of washing the subject's face and walking. We consider the level as an offset level to recognize behavior and diseases. The levels are added according to daily behavior. If there was not an event to increase odor grade, the level was almost constant. The average, standard deviation and coefficient of variation of three sensor outputs at awakening and after walking are summarized in Table 2. The data for sensors 1 and 2 are similar. The data for sensor 3 are different from sensors 1 and 2 . These characteristics correspond to the data on Table 1. There is a tendency for the odor grade after walking to become lower than the grade at awakening. It is effective to compare the breath odor grades for other behavior with this grade.

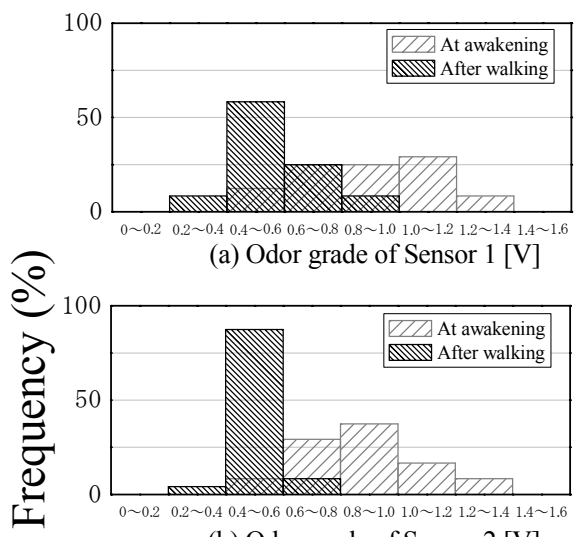

(b) Odor grade of Sensor 2 [V]

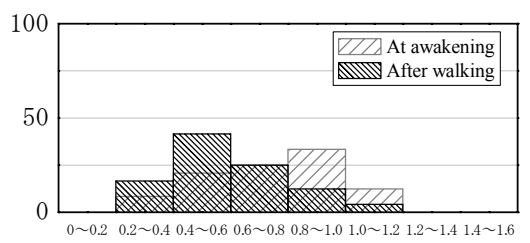

(c) Odor grade of Sensor 3 [V]

Figure 8: Histograms of sensor outputs at awakening and after walking in the morning.

Table 2: Average (ave), standard deviation $(s)$ and coefficient of variation $(c v)$ of the three sensor outputs at awakening and after walking in the morning.

\begin{tabular}{c|c|c|c|c|c|c}
\hline \multirow{2}{*}{} & \multicolumn{3}{|c|}{ At awakening } & \multicolumn{3}{c}{ After walking } \\
\cline { 2 - 7 } & $\mathrm{S} 1$ & $\mathrm{~S} 2$ & $\mathrm{~S} 3$ & $\mathrm{~S} 1$ & $\mathrm{~S} 2$ & $\mathrm{~S} 3$ \\
\hline ave & 0.90 & 0.87 & 0.75 & 0.57 & 0.52 & 0.59 \\
$s$ & 0.22 & 0.21 & 0.28 & 0.15 & 0.10 & 0.22 \\
$c v$ & 25 & 25 & 28 & 26 & 18 & 38 \\
\hline
\end{tabular}




\subsection{The sensor output after brushing teeth}

Brushing teeth is an autonomic human behavior. The behavior is necessary to keep a keen sense in the mouth and to prevent tooth decay and periodontal disease. It is also said that brushing teeth activates cerebral responses that stimulate the gums. It is very important for a person to have tasty meals throughout life, and brushing teeth is effective against the items. In this study, we identified brushing teeth as an important behavior using the odor sensor grades. Polishing materials, medicinal materials and menthol as a flavoring are contained in toothpastes. Consequently, the odor sensor system can detect teeth brushing by sensing menthol. The output characteristics of the odor sensors for brushing teeth are indicated in Figure 9. The output of sensor 2 is larger than that for sensor 1 after brushing. The system can identify brushing teeth from the sensor outputs, and the identification probability is over $90 \%$.

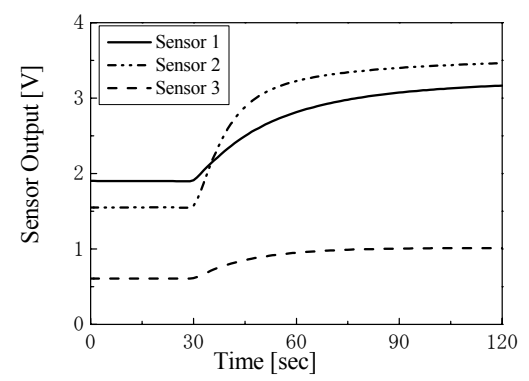

Figure 9: Characteristics of the three odor sensors for exhaled breath after brushing teeth.

\subsection{Identification of eating fruit}

For human beings, fruits have an important role in keeping humans healthy. Therefore, the Ministry of Agriculture, Forestry and Fisheries of Japan recommends the eating of fruit. Apples and oranges are very popular among Japanese because large amounts are harvested in Japan. Apples relieve physical fatigue and also to remove mental fatigue. They are a staple in the prevention of hyperlipidemia.

Oranges contain vitamin $\mathrm{C}$ and some mineral components. People sometimes bleed from the gums and the eyes when they are deficient in vitamin C. Oranges are good to prevent colds, aging, diabetes and heart disease. The breath odor characteristics were examined when a subject ate an apple, an orange and a Hassaku orange. Examples of the results are shown in Figure 10. Figure (a) shows the characteristics for apple, (b) for orange and (c) for Hassaku orange. In these figures, the output of sensor 2 is higher than that of sensor 1 . 


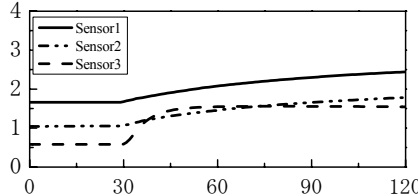

(a) Apple

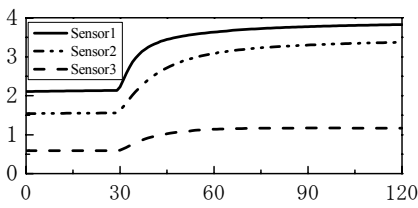

(b) Orange

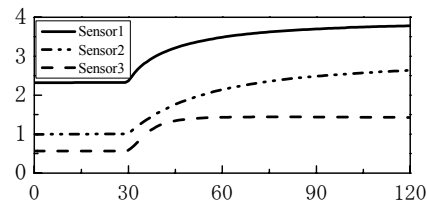

(c) Hassaku orange

Time [sec]

Figure 10: Characteristics of the three odor sensors for exhaled breath after eating fruit: (a) apple, (b) orange and (c) Hassaku orange. Vertical axis means sensor output [V].

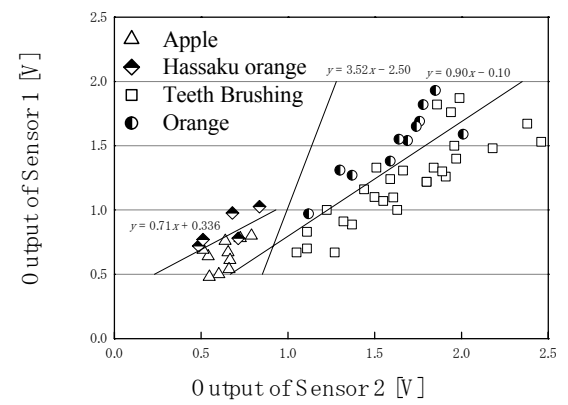

Figure 11: Relationship between sensor 1 and 2 outputs.

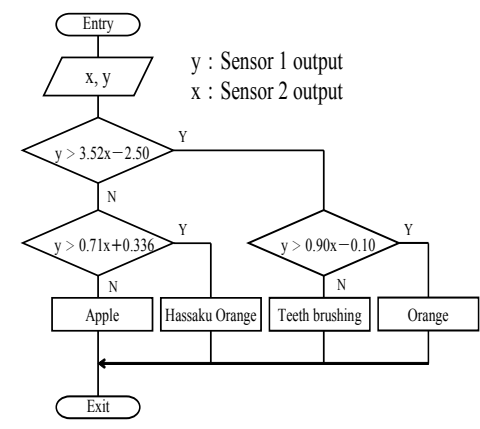

Figure 12: Flow chart for identifying teeth brushing and eating apples, oranges or Hassaku oranges.

\subsection{Identification of fruit and teeth brushing using a scatter diagram}

The identification of the eating of an apple, an orange and a Hassaku orange and teeth brushing as behavior is investigated using the three odor sensor outputs. The system adopted the outputs of three sensors when the identification was carried out. An example of a scatter diagram is shown in Figure 11. The figure shows the scatter diagram for sensors $1(y)$ and $2(x)$ when the subject has eaten fruit and brushed teeth. We can distinguish the apple-Hassaku orange group from the orange-teeth brushing group using a discriminant function, and the identification rate is $100 \%$. The discriminant function derived is shown in eq. (1), in which $y$ means sensor 1 output and $x$ means sensor 2 output.

$$
y=3.52 x-2.50
$$


We can also distinguish the orange group from the teeth brushing group using a discriminant function, but the identification rate in that case is $85 \%$. The function is indicated in eq. (2). The system can also distinguish the apple group from the Hassaku orange group using the same function, and the identification rate is over $90 \%$. The function is shown in eq. (3). It is, however, better to gather more data to confirm these relationship.

$$
\begin{aligned}
& y=0.90 x-0.10 \\
& y=0.71 x+0.336
\end{aligned}
$$

The flow chart for the identification is shown in Figure 12. In the first place, the outputs $(x, y)$ are entered into the system. The apple-Hassaku orange group and orange-teeth brushing group are classified. Then "orange" and "teeth brushing" are separated. After that, "apple" and "Hassaku orange" are separated.

\subsection{Identification of stress and fatigue}

Stress and fatigue are spread among human beings according to the progress of IT society. It is sometimes said that mental fatigue occurs more frequently than physical fatigue in the elderly population. The following conditions are caused by fatigue; headache, high blood pressure, angina and some intraoral diseases. If a person has intraoral diseases, the inside of the mouth dries and bad breath odor results. In this experiment, first the subject identified the grade of stress and fatigue due to bodily sensations. The grades were expressed in five stages. Sensor 3 output was then examined. The results are shown in Figure 13. Sensor 3 output, which is highly sensitivity to hydrogen gas, increases as the grade of mental and physical fatigue ( $r m f, r p f)$ based on bodily sensations of the subject increases. The output also became markedly higher when the subject had a cold and took a medicine. In this experiment, the system achieved a high correlation coefficient of about 0.9 (S3). There was dispersion in the data between the sensor output and the grade of fatigue.

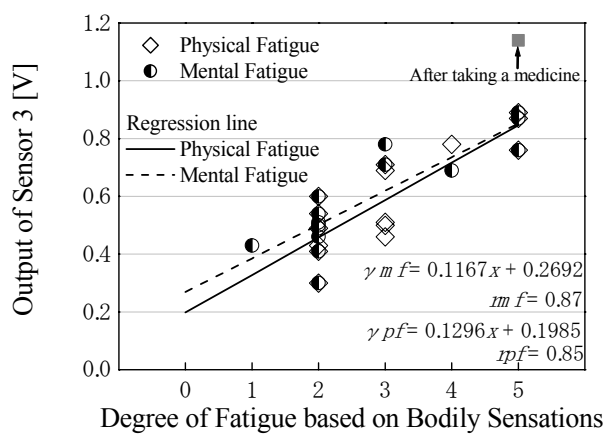

Figure 13: Correlation between degree of fatigue based on bodily sensations and output of sensor 3 . 


\section{Conclusion}

The pattern of life in the elderly and the grade of fatigue were examined by investigating breath odor using three metal oxide gas sensors. These sensors have different sensitivities to chemical substances. Sensor 3, an indium oxide type, has high sensitivity to hydrogen gas. The volume of hydrogen gas in exhaled breath increases when the subject is fatigued. The sensor outputs regarding breath odor show fluctuations in daily patterns. The patterns arise according to eating meals regularly. The odor after walking in the morning is lower than the one at awakening. Brushing teeth, which is an autonomous behavior, and eating three kinds of citrus fruits can be identified. The output level of sensor 3 is linear with respect to the grade of fatigue based on subjective bodily sensations. As sensor 3 is highly sensitivity to hydrogen gas, this observation supports the idea that hydrogen concentration in exhaled breath increases when the subject is fatigued.

The pattern of life in the elderly may be surveyed in detail without the invasion of privacy, and a survey system with high reliability for the aged may be constructed.

\section{References}

[1] Diamond, D., Principles of Chemical and Biological Sensors, New York, John Wiley \& Sons, 1998.

[2] Gardner, J.W. \& Bartlett, P.N., Electronic Noses Principles and Applications, Oxford, Oxford University Press, 1999.

[3] Gardner, J.W., Microsensors, New York, John Wiley \& Sons, 1994.

[4] Joshi, R. \& Sanderson, A.C., Multisensor Fusion, New Jersey, World Scientific, 1999.

[5] Mutambara, G.O., Decentralized Estimation and Control for Multisensor Systems, Boston, CRC Press, 1998.

[6] Oyabu, T., Okada, A., Manninen, O. \& Lee, D.-D., Proposition of a survey device with odor sensors for an elderly person. Sensors \& Actuators B, 96, pp.239-244, 2003. 